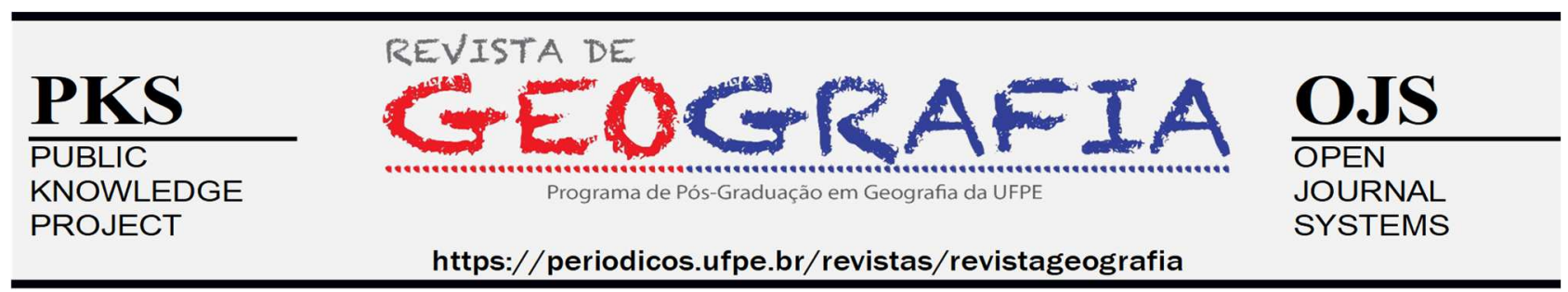

\title{
EVOLUÇÃO DA CONTAMINAÇÃO POR METAIS PESADOS EM SEDIMENTOS EM ÁREA ESTUARINA DO RIO CAPIBARIBE: UMA REVISÃO
}

\begin{abstract}
João Carlos Morais de Araújo Júnior ${ }^{1}$, Ricardo Pereira ${ }^{2}$
${ }^{1}$ Mestre em Geociências. Universidade Federal de Pernambuco. E-mail: jcmorais.araujo@gmail.com. ORCID: http://orcid.org/0000-0001-5392-9831.

${ }^{2}$ Professor do Departamento de Geologia e no Programa de Pós-Graduação em Geociências. Universidade Federal de Pernambuco. E-mail: ricardo.pereira2@ufpe.br. ORCID: http://orcid.org/0000-0002-7737-3206

Artigo recebido em 14/09/2020 e aceito em 03/03/2021

RESUMO

A região estuarina do rio Capibaribe vem a sofrer modificações no seu meio físico e químico devido à intensificação das ações antrópicas que interferem na dinâmica estuarina, ocasionando tanto a destruição na paisagem, quanto a adição de compostos e elementos tóxicos que prejudicam a biota local. Devido à rápida degradação da qualidade ambiental deste estuário, este trabalho apresenta uma revisão sobre a evolução das concentrações de metais pesados em sedimentos, com base em dados disponíveis na literatura nos últimos 20 anos. As pesquisas realizadas indicam que a região estuarina está contaminada por metais pesados (Al, Ag, As, Cd, Co, Cr, Cu, Fe, Hg, Mn, Mg, Ni, Pb, Zn). Particularmente os metais $\mathrm{Ag}, \mathrm{Cu}, \mathrm{Pb}$ e $\mathrm{Zn}$ possuem concentrações acima dos limites de segurança estabelecidos pelas agências internacionais e trazem efeitos adversos para a biota local. Os sedimentos localizados próximos ao centro do Recife são os mais impactados para a região do estuário do rio Capibaribe. Dessa maneira, existe a necessidade urgente de mais pesquisas quanto à origem desses metais e ações que busquem a remediação do ambiente contaminado e previnam mais impactos ao ecossistema representado pelo estuário do rio Capibaribe.
\end{abstract}

Palavras-chave: rio Capibaribe. Estuário. Metais pesados. Contaminantes. Sedimentos.

\section{EVOLUTION OF CONTAMINATION BY HEAVY METALS IN SEDIMENTS IN ESTUARINE AREA OF RIO CAPIBARIBE: A REVIEW}

\begin{abstract}
The estuarine region of the Capibaribe River has changed its physical and chemical environment due to the intensification of the anthropic actions that interfere with the estuarine dynamics, causing both the destruction in the landscape, as well as the addition of compounds and toxic elements that harm the local biota. Due to the rapid degradation of the environmental quality of this estuary, this work presents a review of the evolution of concentrations of heavy metals in sediments, based on data available in the literature in the last 20 years. The research carried out indicates that the estuarine region is contaminated by heavy metals ( $\mathrm{Al}, \mathrm{Ag}, \mathrm{As}, \mathrm{Cd}, \mathrm{Co}, \mathrm{Cr}, \mathrm{Cu}, \mathrm{Fe}, \mathrm{Hg}, \mathrm{Mn}, \mathrm{Mg}, \mathrm{Ni}, \mathrm{Pb}, \mathrm{Zn}$ ). Particularly the metals $\mathrm{Ag}, \mathrm{Cu}, \mathrm{Pb}$, and $\mathrm{Zn}$ have concentrations above the safety limits established by international agencies and bring adverse effects to the local biota. The sediments located near the center of Recife are the most impacted for the region of the Capibaribe River estuary. Thus, there is an urgent need for more research on the origin of these metals and actions that seek to remedy the contaminated environment and prevent further impacts to the ecosystem represented by the Capibaribe River estuary.
\end{abstract}

Keywords: Capibaribe river. Estuary. Heavy metals. Contaminants. Sediments. 


\section{INTRODUÇÃO}

O estuário do rio Capibaribe está localizado na Região Nordeste do Brasil e vem recebendo impacto da ação antrópica desde o século XVII devido ao lançamento de diversos contaminantes, ocasionando um grande nível de poluição ambiental neste ambiente (XAVIER et al., 2017). A bacia hidrográfica do rio Capibaribe abrange uma área de drenagem com cerca de 7.454,88 km² e localizase no estado de Pernambuco, entre as coordenadas geográficas $07^{\circ} 41^{\prime} 20^{\prime \prime}$ e $08^{\circ} 19^{\prime} 30^{\prime \prime}$ de latitude

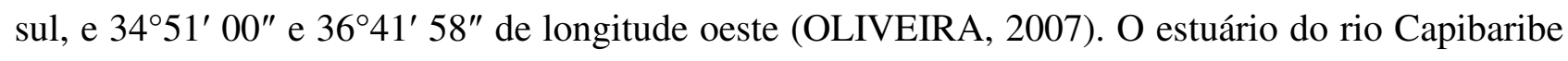
está inserido em área de zona urbana, incorporando os municípios de São Lourenço da Mata, Camaragibe e Recife, sendo constituído pelos rios Capibaribe, Tejipió, Jordão e Pina (SANTOS et al., 2009). Devido ao processo de industrialização e a ocupação urbana, o rio Capibaribe tem sido motivo de preocupação devido às atividades antrópicas relacionadas com os lançamentos de efluentes industriais e domésticos, sendo evidentes as alterações ambientais causadas pela urbanização em sua proximidade, particularmente na Região Metropolitana do Recife (NÓBREGA, 2011; SILVA et al., 2010; SILVA, 2004).

Os estuários são corpos d'água semifechados, localizados em áreas de transição entre o continente e o oceano, sendo caracterizados como um ecossistema dinâmico de grande produtividade e diversidade, o que os tornam indispensáveis para a preservação de vários organismos (BIRCH; OLMOS, 2008; CUNHA; PINTO; DINIS, 1997; CICIN-SAIN; KNECHT, 1998). Comumente, as regiões estuarinas são importantes fontes de recursos naturais que podem ser utilizadas como fonte de alimento, sustento e lazer pela população. Normalmente as grandes cidades e complexos industriais estão próximos das áreas estuarinas e frequentemente liberam quantidades significativas de rejeitos orgânicos e inorgânicos sem o devido tratamento, o que acarreta consequências negativas à biota, à saúde e para as atividades humanas (CUNHA et al., 1997; LIU et al., 2003; SANTOS et al. 2009; VERONEZ JUNIOR et al., 2009).

A maior parte dos efluentes liberados em corpos hídricos possuem substâncias com características hidrofóbicas, apresentando baixa solubilidade, sendo adsorvidos em sedimentos e podendo ser absorvidos por organismos (YANG et al., 2012). Sua composição abrange diversos contaminantes, como petróleo e seus derivados, organoclorados, fármacos, nutrientes em excesso e, particularmente, metais pesados (MACIEL, 2015; CAJARAVILLE et al., 2000). As quantidades aumentadas desses metais ocasionam concentrações tóxicas nos compartimentos aquáticos, terrestres e atmosféricos. Com isso a população pode sofrer exposição e ocorrer a acumulação desses elementos 
em seu organismo provocando diversos problemas de saúde, como anemia, câncer ou alteração no sistema nervoso central (AZEVEDO; CHASIN, 2003).

Os metais pesados também são inseridos no ambiente aquático através de fontes naturais, como consequência do intemperismo de rochas e solos, além das fontes antrópicas como resíduos provenientes da agricultura, esgotos domésticos e industriais (CEVIK et al., 2009). Atualmente a contaminação por metais pesados no meio ambiente é considerada como um sério problema mundial, devido à sua elevada toxicidade e seu fator de acumulação em organismos (LIU et al., 2015). De acordo com Duffus (2002), metais pesados são definidos como elementos com massa específica entre 3,5 e 7,0 g.cm-3 e número atômico maior que 20. Além disso, o conceito de metais pesados pode ser mais abrangente devido ao impacto ao meio ambiente e à saúde humana resultante de seu descarte. Sendo assim, fatores ambientais, geoquímicos e toxicológicos podem ser empregados para uma melhor definição do que seria um metal pesado, considerando biodisponibilidade versus geodisponibilidade, bem como os efeitos adversos decorrentes da toxicidade da espécie química analisada. Devido ao seu alto grau de toxicidade, diferentes cargas iônicas e capacidade de formar complexos orgânicos, os elementos $\mathrm{Hg}, \mathrm{Cd}, \mathrm{Cu}, \mathrm{Pb}, \mathrm{Mn}, \mathrm{Tl}, \mathrm{Cr}, \mathrm{Ni}, \mathrm{Se}, \mathrm{Sb}, \mathrm{Be}, \mathrm{Co}, \mathrm{Mo}, \mathrm{Sn}, \mathrm{W}$ e V estão entre os mais estudados (MORAES; SZNELWAR; FERNÍCOLA, 1991; VALLS; LORENZO, 2002; TAVARES; CARVALHO, 1992; WOOD, 1974). Esses elementos concentram-se no meio estuarino devido a processos de floculação, adsorção em partículas orgânicas, inorgânicas e posteriormente ocorrendo à sedimentação (LIU et al., 2009).

A análise dos sedimentos é de grande importância, pois reflete a poluição de rios e estuários por metais pesados, além de relacionar a forma química, a mobilidade do elemento e a concentração do metal no sistema aquático (WANG et al., 2017; GU et al., 2015; LIMA et al., 2007). Por isso são utilizados para identificar a presença de contaminantes que não permanecem solúveis após o seu lançamento em águas superficiais. A mobilidade e a forma química dos metais pesados são influenciadas principalmente por fatores físico-químicos e geológicos, como o potencial hidrogeniônico $(\mathrm{pH})$, potencial redox (Eh) e a granulometria dos sedimentos (GU et al., 2015).

A granulometria e a composição do sedimento influenciam na capacidade de ligação dos contaminantes, pois refletem as condições físico-químicas do ambiente em relação à remobilização, erosão, sedimentação e capacidade de adsorção (DEVESA-REY et al., 2011). As frações silte, argila e matéria orgânica elevam a capacidade de reter os íons metálicos por meio da adsorção, quelação e troca iônica (WILLIAMS; BUBB; LESTER, 1994). De acordo com French (1997) a adsorção de 
metais pesados através dos argilominerais, matéria orgânica ou hidróxidos de ferro/manganês possuem grande importância devido à deposição desses elementos químicos no ambiente estuarino.

Através de mudanças físico-químicas no ambiente, envolvendo parâmetros como $\mathrm{pH}$, potencial redox e salinidade, os metais pesados imobilizados nos sedimentos podem ser mobilizados para o ambiente aquático, desse modo, os sedimentos atuam como uma fonte secundária de contaminação (SALOMONS; FORSTNER, 1984). A análise da qualidade dos sedimentos é recomendada para o entendimento da disponibilidade desses elementos químicos no ambiente, bem como o grau de contaminação e a possível origem (WANG et al., 2017; ALMEIDA; ROCHA, 2006).

O objetivo deste trabalho foi realizar uma revisão quanto a contaminação por metais pesados no estuário do rio Capibaribe, condensando e analisando as principais informações disponíveis na literatura. Com isso, pode-se efetuar uma avaliação quanto à evolução dos níveis de metais pesados na região estuarina, reunindo informações que abrangem a contaminação por metais pesados de 1812 até 2015. Pretende-se com isso reunir dados quanto às concentrações dos metais pesados presentes no estuário do rio Capibaribe ao longo de dois séculos e sua relação com as possíveis fontes existentes na Região Metropolitana do Recife.

\section{Caracterização do estuário do rio Capibaribe}

O Brasil dispõe de uma ampla região costeira que está localizada em uma faixa que se estende por mais de 8.500 km em direção ao Oceano Atlântico (MMA, 2019). Diversos ecossistemas estão presentes na costa brasileira, como os estuários e manguezais que estão dispostos desde a região Norte do país, localizado no estado do Amapá, alcançando o Sul do país, no estado de Santa Catarina (SCHAEFFER et al., 1990).

Os estuários localizados no Nordeste brasileiro são sensíveis às variações do nível do mar e a mudanças do regime fluvial, sendo associado às grandes amplitudes das marés e às baixas superfícies das bacias costeiras que resultam em uma intrusão salina no continente (LACERDA et al., 2008).

O estado de Pernambuco possui uma das mais estreitas faixas litorâneas da costa brasileira, tendo poucas regiões estuarinas. Estas ocupam cerca de 25.040 hectares, dos quais 17.372 hectares são formados de vegetação de mangue (COELHO; TORRES, 1982; BARROS et al., 2000). No litoral pernambucano existem cerca de quinze estuários, dentre eles, o sistema estuarino do rio Capibaribe, sendo o principal contribuinte de água doce abrangendo uma bacia de drenagem com cerca de 7.500 
$\mathrm{km}^{2}$. Esta região também inclui a Bacia do Pina que compreende um corpo de água quase paralelo ao canal do estuário e contém vários afluentes, como os rios Jordão, Pina e Tejipió (Figura 1). Desta forma, o sistema estuarino do rio Capibaribe atua como uma rede de drenagem urbana da Região Metropolitana do Recife, trazendo efluentes não tratados originados de atividades industriais e domésticas para o sistema estuarino que são provenientes de diversos municípios (SANTOS et al., 2009). O regime de marés que ocorre neste sistema estuarino pode ser classificado como sendo do tipo semi-diurno alcançando alturas de 1,5 e 3 metros, respectivamente, no período de quadratura e sizígia. O regime hidrológico anual é determinado com o período de chuvas nos meses de março a agosto e pelo período seco entre setembro e fevereiro (FEITOSA; NASCIMENTO; COSTA, 1999).

FIGURA 1 - LOCALIZAÇÃO GEOGRÁFICA DO ESTADO DE PERNAMBUCO NO NORDESTE DO BRASIL E DO ESTUÁRIO DO RIO CAPIBARIBE, DETALHADOS PELA FOTOGRAFIA AÉREA DE IMAGEM DE SATÉLITE. 1 - RIO CAPIBARIBE; 2 - BRAÇO SUL DO RIO CAPIBARIBE; 3 - RIO TEJIPIÓ/JIQUIÁ; 4 - RIO JORDÃO; 5 - RIO PINA; 6 - BACIA DO PINA; I - PARQUE DOS MANGUEZAIS; II - PORTO DO RECIFE; *VIVEIRO DE PEIXE E CAMARÃO.

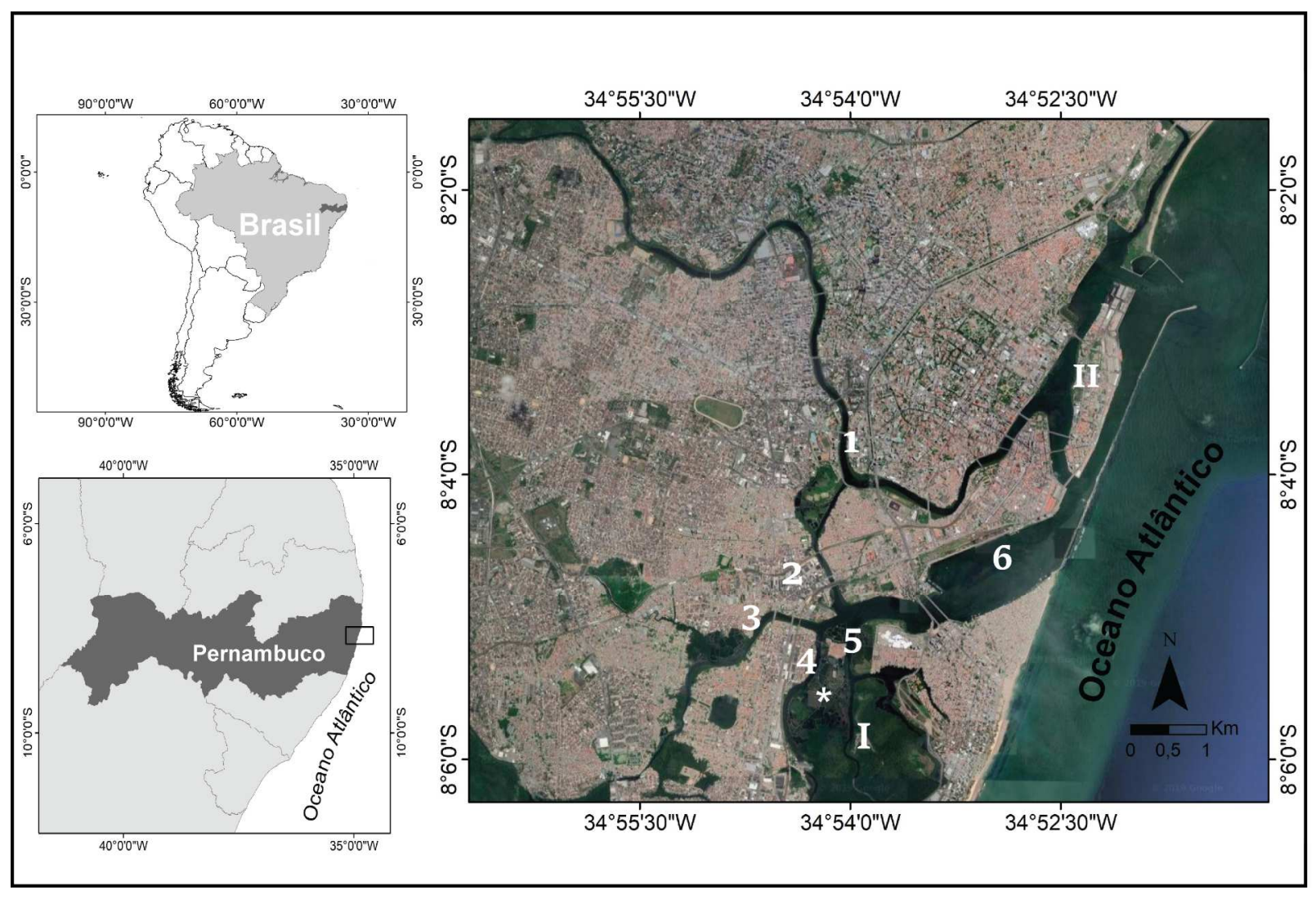

Fonte: Elaborado pelos autores (2020).

O padrão sedimentológico do sistema estuarino do rio Capibaribe apresenta diferenças em relação aos períodos seco e chuvoso. A época seca é marcada por uma sedimentação de granulometria 
variando de silte muito fino a areia grossa, enquanto no período chuvoso é registrada uma granulometria variando de argila a areia média, com predominância de silte muito fino (OLIVEIRA et al., 2014). O estuário do rio Capibaribe é caracterizado como um ambiente deposicional de baixa energia, dominado pela influência de fontes terrígenas e antrópicas, além da ação marinha, possuindo composição siliciclástica e argilosa, com alta concentração de matéria orgânica de origem mista, porém tendendo a uma fonte continental (OLIVEIRA et al., 2014).

\section{MATERIAIS E MÉTODOS}

A compilação de dados referentes à presença de metais pesados na região estuarina do rio Capibaribe foi realizada por consultas a monografias, dissertações, teses e artigos, reunindo-se assim as principais informações disponíveis sobre a presença de metais pesados (Al, Ag, As, Cd, Co, Cr, $\mathrm{Cu}, \mathrm{Fe}, \mathrm{Hg}, \mathrm{Mn}, \mathrm{Mg}, \mathrm{Ni}, \mathrm{Pb}, \mathrm{Zn}$ ) no estuário. Foram utilizados SciElo, Scopus® e Science Direct, bem como portais de bibliotecas de diversas universidades federais. Como resultado, foram levantadas 12 referências bibliográficas que reportam a análise de metais pesados em sedimentos na região estuarina do rio Capibaribe. Com isso, foram reunidas todas as referências bibliográficas disponíveis na literatura sobre o assunto, representadas pelos trabalhos de Brayner (1998); Brayner, Barbosa e Silva (2001); Brayner et al. (2003); Silva (2004); Macedo et al. (2007); Silva et al. (2010); Maciel (2015); Régis (2016); Xavier (2017); Xavier et al. (2017); Barcellos et al. (2017) e Régis et al. (2018).

Os dados das distribuições dos pontos de coleta de sedimentos foram compilados e organizados, posteriormente, utilizou-se a base cartográfica de arquivos vetoriais disponíveis na página eletrônica do Instituto Brasileiro de Geografia e Estatística (IBGE) e através do software QGis 2.18 foram confeccionados os mapas de localização e distribuição dos pontos de monitoramento ao longo dos anos no estuário do rio Capibaribe.

\section{RESULTADOS E DISCUSSÃO}

Os trabalhos realizados no estuário do rio Capibaribe analisaram principalmente sedimentos, caracterizando a presença de metais pesados e compostos como hidrocarbonetos, organoclorados e toxicidade (MACIEL, 2015; MACIEL et al., 2015; SILVA, 2004; XAVIER, 2017). A figura 2 e o quadro 1, respectivamente, apresentam um mapa com a localização dos pontos de amostragem de sedimentos e os elementos químicos relatados pelos autores nesta revisão. 
FIGURA 2 - MAPA DO ESTUÁRIO DO RIO CAPIBARIBE, APRESENTANDO AS LOCALIZAÇÕES DOS PONTOS DE COLETA. 1 - RIO CAPIBARIBE; 2 - BRAÇO SUL DO RIO CAPIBARIBE; 3 - RIO TEJIPIÓ/JIQUIÁ; 4 - RIO JORDÃO; 5 - RIO PINA; 6 - BACIA DO PINA; I - PARQUE DOS MANGUEZAIS; II - PORTO DO RECIFE.

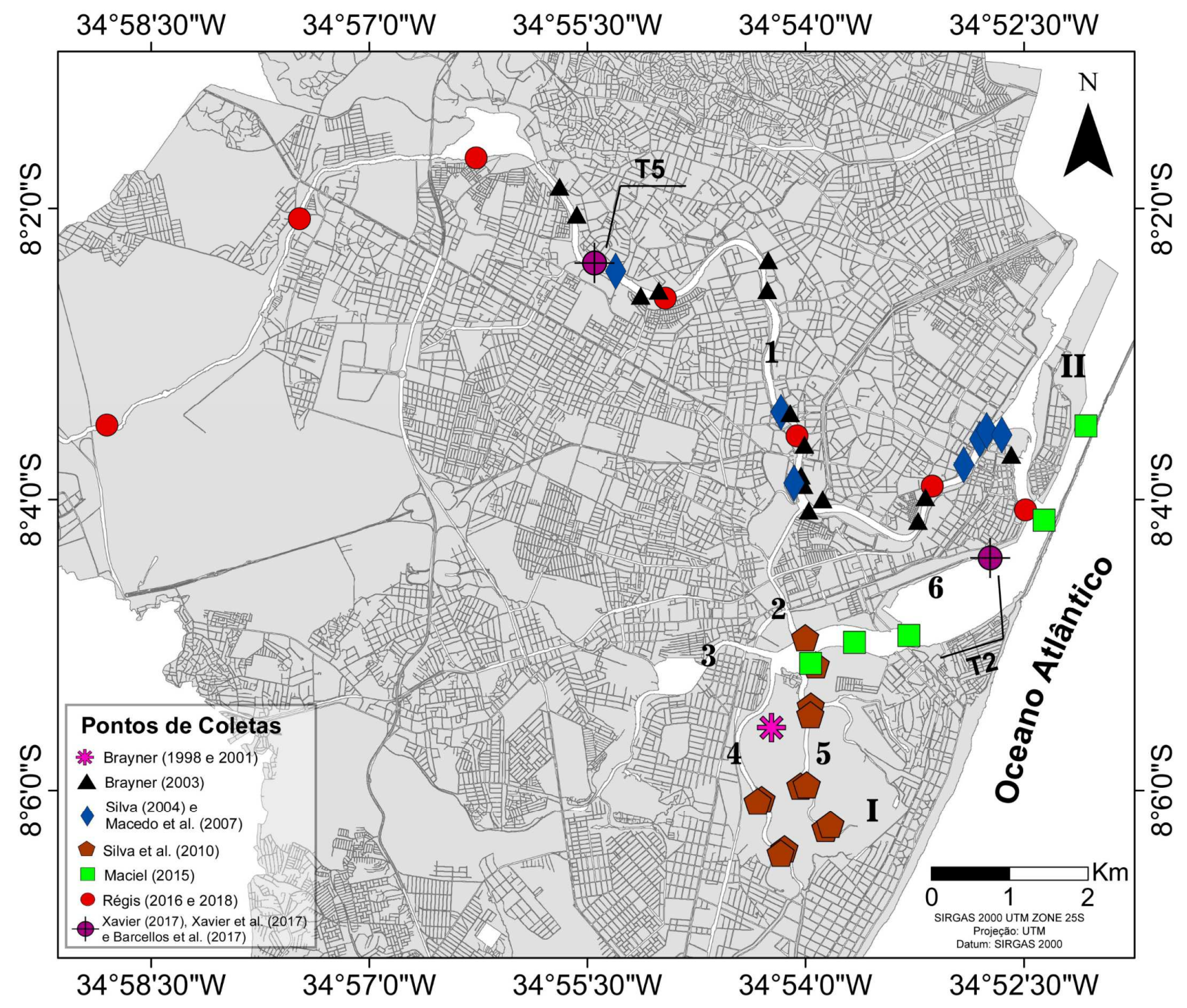

Fonte: Elaborado pelos autores (2020). 
QUADRO 1 - ANÁLISES DE SEDIMENTOS DO ESTUÁRIO DO RIO CAPIBARIBE.

\begin{tabular}{|c|c|c|c|}
\hline Metais Pesados & $\begin{array}{c}\text { Considerações dos } \\
\text { autores }\end{array}$ & $\begin{array}{l}\text { Período de } \\
\text { coleta }\end{array}$ & Referência \\
\hline $\begin{array}{c}\mathrm{Cd}\left(2,9-13,12 \mathrm{mg} \cdot \mathrm{kg}^{-1}\right) \\
\mathrm{Co}\left(9,38-17,71 \mathrm{mg} \cdot \mathrm{kg}^{-1}\right) \\
\mathrm{Cu}\left(31,64-131,58 \mathrm{mg} \cdot \mathrm{kg}^{-1}\right) \\
\mathrm{Mg}\left(33,5-311,94 \mathrm{mg} \cdot \mathrm{kg}^{-1}\right) \\
\mathrm{Ni}\left(12,47-39,56 \mathrm{mg} \cdot \mathrm{kg}^{-1}\right) \\
\mathrm{Pb}\left(5,582-163,38 \mathrm{mg} \cdot \mathrm{kg}^{-1}\right) \\
\mathrm{Zn}\left(82,67-184,55 \mathrm{mg} \cdot \mathrm{kg}^{-1}\right)\end{array}$ & $\begin{array}{l}\text { Concentrações totais no } \\
\text { sedimento superficial }\end{array}$ & 2001 e 2002 & $\begin{array}{l}\text { Brayner et al. } \\
\qquad(2003)\end{array}$ \\
\hline $\begin{array}{c}\mathrm{Cd}\left(16-242 \mathrm{mg} \cdot \mathrm{kg}^{-1}\right) \\
\mathrm{Cr}\left(76-247 \mathrm{mg} \cdot \mathrm{kg}^{-1}\right) \\
\mathrm{Cu}\left(21-146 \mathrm{mg} \cdot \mathrm{kg}^{-1}\right) \\
\mathrm{Fe}\left(31.080-52.013 \mathrm{mg} \cdot \mathrm{kg}^{-1}\right) \\
\mathrm{Mn}\left(173-465 \mathrm{mg} \cdot \mathrm{kg}^{-1}\right) \\
\mathrm{Zn}\left(144-406 \mathrm{mg} \cdot \mathrm{kg}^{-1}\right)\end{array}$ & $\begin{array}{l}\text { Sedimento superficial. } \\
\text { Apresentam níveis de } \\
\text { concentração acima do } \\
\text { valor obtido de } \\
\text { Background, indicando } \\
\text { contaminação (Fe, Mn, Zn, } \\
\text { Cu e Cd) }\end{array}$ & $\begin{array}{l}\text { Julho a } \\
\text { agosto } \\
(2002)\end{array}$ & $\begin{array}{c}\text { Silva (2004) } \\
\mathrm{e}\end{array}$ \\
\hline $\begin{array}{c}\mathrm{Cd}\left(<16-167 \mathrm{mg} \cdot \mathrm{kg}^{-1}\right) \\
\mathrm{Cr}\left(87-157 \mathrm{mg} \cdot \mathrm{kg}^{-1}\right) \\
\mathrm{Cu}\left(12-136 \mathrm{mg} \cdot \mathrm{kg}^{-1}\right) \\
\mathrm{Fe}\left(36.053-48.880 \mathrm{mg} \cdot \mathrm{kg}^{-1}\right) \\
\mathrm{Mn}\left(154-391 \mathrm{mg} \cdot \mathrm{kg}^{-1}\right) \\
\mathrm{Zn}\left(191-655 \mathrm{mg} \cdot \mathrm{kg}^{-1}\right) \\
\end{array}$ & $\begin{array}{l}\text { Material Sedimentado. } \\
\text { Possuem concentrações } \\
\text { maiores que os sedimentos } \\
\text { superficiais } \\
\text { Possuem concentração } \\
\text { acima do Background local }\end{array}$ & $\begin{array}{c}\mathrm{e} \\
\text { Janeiro e } \\
\text { Fevereiro } \\
(2003)\end{array}$ & $\begin{array}{l}\text { Macedo et al. } \\
\text { (2007) }\end{array}$ \\
\hline $\begin{array}{c}\mathrm{Cr}\left(25-231 \mathrm{mg} \cdot \mathrm{kg}^{-1}\right) \\
\mathrm{Fe}\left(11.500-43.120 \mathrm{mg} \cdot \mathrm{kg}^{-1}\right) \\
\mathrm{Mg}\left(91-338 \mathrm{mg} \cdot \mathrm{kg}^{-1}\right) \\
\mathrm{Zn}\left(75-613 \mathrm{mg} \cdot \mathrm{kg}^{-1}\right)\end{array}$ & $\begin{array}{l}\text { Concentrações acima do } \\
\text { Background, área } \\
\text { contaminada } \\
(\mathrm{Cr}, \mathrm{Mg} \text { e } \mathrm{Zn})\end{array}$ & $\begin{array}{l}\text { Novembro } \\
\text { (2006) }\end{array}$ & $\begin{array}{l}\text { Silva et al. } \\
\quad(2010)\end{array}$ \\
\hline $\begin{array}{c}\mathrm{Al}\left(1,2-2,3 \mu \mathrm{g} \cdot \mathrm{g}^{-1}\right) \\
\mathrm{Cd}\left(0,1-0,4 \mu \mathrm{g} \cdot \mathrm{g}^{-1}\right) \\
\mathrm{Cr}\left(31,0-58,0 \mu \mathrm{g} \cdot \mathrm{g}^{-1}\right) \\
\mathrm{Fe}\left(1,5-2,6 \mu \mathrm{g} \cdot \mathrm{g}^{-1}\right) \\
\mathrm{Hg}\left(0,1-0,3 \mu \mathrm{g} \cdot \mathrm{g}^{-1}\right) \\
\mathrm{Ni}\left(10,6-17,2 \mu \mathrm{g} \cdot \mathrm{g}^{-1}\right) \\
\mathrm{Pb}\left(17,7-35,8 \mu \mathrm{g} \cdot \mathrm{g}^{-1}\right) \\
\mathrm{Zn}\left(96,0-275,0 \mu \mathrm{g} \cdot \mathrm{g}^{-1}\right)\end{array}$ & $\begin{array}{c}\mathrm{Cu}, \mathrm{Pb} \text { e } \mathrm{Zn} \text { apresentam } \\
\text { concentrações acima do } \\
\text { "ERL", } \\
\text { porém valores abaixo do } \\
\text { "ERM". Contudo para } \mathrm{Cu}, \\
\mathrm{Pb} \text { e } \mathrm{Zn} \text { apresentam } \\
\text { concentrações superiores ao } \\
\text { ERM e apresentam efeitos } \\
\text { adversos à biota local. }\end{array}$ & $\begin{array}{l}\text { Setembro e } \\
\text { Dezembro } \\
(2011)\end{array}$ & $\begin{array}{l}\text { Maciel } \\
(2015)\end{array}$ \\
\hline
\end{tabular}


QUADRO 1 - ANÁLISES DO SEDIMENTO DO ESTUÁRIO DO RIO CAPIBARIBE (CONTINUAÇÃO).

\begin{tabular}{|c|c|c|c|}
\hline Metais Pesados & $\begin{array}{c}\text { Considerações dos } \\
\text { autores }\end{array}$ & $\begin{array}{c}\begin{array}{c}\text { Período de } \\
\text { coleta }\end{array} \\
\end{array}$ & Referência \\
\hline $\begin{array}{c}\mathrm{Ag}\left(<0,01-2,96 \mu \mathrm{g} \cdot \mathrm{g}^{-1}\right) \\
\text { As }\left(<1-10 \mu \mathrm{g} \cdot \mathrm{g}^{-1}\right) \\
\mathrm{Cd}\left(0,07-0,69 \mu \mathrm{g} \cdot \mathrm{g}^{-1}\right) \\
\mathrm{Cr}\left(32-66 \mu \mathrm{g} \cdot \mathrm{g}^{-1}\right) \\
\mathrm{Cu}\left(17,10-80,50 \mu \mathrm{g} \cdot \mathrm{g}^{-1}\right) \\
\mathrm{Hg}\left(0,02-0,61 \mu \mathrm{g} \cdot \mathrm{g}^{-1}\right) \\
\mathrm{Ni}\left(11,60-23,30 \mu \mathrm{g} \cdot \mathrm{g}^{-1}\right) \\
\mathrm{Pb}\left(16,30-41,70 \mu \mathrm{g} \cdot \mathrm{g}^{-1}\right) \\
\mathrm{Zn}\left(53-306 \mu \mathrm{g} \cdot \mathrm{g}^{-1}\right)\end{array}$ & $\begin{array}{c}\mathrm{Cd}, \mathrm{Cr}, \mathrm{Cu}, \mathrm{Ni}, \mathrm{Pb} \\
\text { encontram-se em níveis } \\
\text { maiores que o "TEL", } \\
\text { porém concentrações } \\
\text { abaixo do nível do "PEL", } \\
\text { enquanto os elementos Ag } \\
\text { e Zn encontram-se em } \\
\text { níveis superiores ao "PEL" }\end{array}$ & $\begin{array}{c}\text { Outubro } \\
\text { (2014) } \\
\text { e } \\
\text { Maio } \\
\text { (2015) }\end{array}$ & $\begin{array}{c}\text { Régis } \\
(2016) \\
\mathrm{e} \\
\text { Régis et al. } \\
\text { (2018) }\end{array}$ \\
\hline $\begin{array}{c}\mathrm{Al}(5,5-14,1 \%) \\
\mathrm{As}\left(19,9-406,7 \mathrm{mg} \cdot \mathrm{kg}^{-1}\right) \\
\mathrm{Co}\left(5,8-22,6 \mathrm{mg} \cdot \mathrm{kg}^{-1}\right) \\
\mathrm{Cu}\left(6,4-83,1 \mathrm{mg} \cdot \mathrm{kg}^{-1}\right) \\
\mathrm{Fe}(1,2-5,1 \%) \\
\mathrm{Mn}\left(183,1-899 \mathrm{mg} \cdot \mathrm{kg}^{-1}\right) \\
\mathrm{Ni}\left(14,1-39,2 \mathrm{mg} \cdot \mathrm{kg}^{-1}\right) \\
\mathrm{Pb}\left(26,6-122,3 \mathrm{mg} \cdot \mathrm{kg}^{-1}\right) \\
\mathrm{Zn}\left(58,8-214,1 \mathrm{mg} \cdot \mathrm{kg}^{-1}\right)\end{array}$ & $\begin{array}{c}\text { Concentrações obtidas para } \\
\text { o testemunho T5, entre os } \\
\text { anos de } 1812 \text { a } 2012 . \\
\text { Foram identificadas quatro } \\
\text { unidades com } \\
\text { características sedimentares } \\
\text { e geoquímica. }\end{array}$ & \multirow{2}{*}{$\begin{array}{c}\text { Novembro } \\
\text { (2012) }\end{array}$} & \multirow{2}{*}{$\begin{array}{l}\text { Xavier (2017); } \\
\text { Xavier et al. } \\
\quad(2017) \\
\text { e } \\
\text { Barcellos et. al. } \\
\quad(2017)\end{array}$} \\
\hline $\begin{array}{c}\mathrm{Al}(3,4-10,7 \%) \\
\mathrm{As}\left(1,6-170,9 \mathrm{mg} \cdot \mathrm{kg}^{-1}\right) \\
\mathrm{Co}\left(4,8-26,6 \mathrm{mg} \cdot \mathrm{kg}^{-1}\right) \\
\mathrm{Cu}\left(52,3-98,3 \mathrm{mg} \cdot \mathrm{kg}^{-1}\right) \\
\mathrm{Fe}(0,9-5.5 \%) \\
\mathrm{Mn}\left(134,5-779,1 \mathrm{mg} \cdot \mathrm{kg}^{-1}\right) \\
\mathrm{Ni}\left(10,7-67,7 \mathrm{mg} \cdot \mathrm{kg}^{-1}\right) \\
\mathrm{Pb}\left(20,4-53,4 \mathrm{mg} \cdot \mathrm{kg}^{-1}\right) \\
\mathrm{Zn}\left(52,5-310,9 \mathrm{mg} \cdot \mathrm{kg}^{-1}\right)\end{array}$ & $\begin{array}{c}\text { Concentrações obtidas para } \\
\text { o testemunho T2 para os } \\
\text { anos de } 1812 \text { a } 2012 . \\
\text { Foram reconhecidas } \\
\text { Três unidades com } \\
\text { características sedimentares } \\
\text { e geoquímica. }\end{array}$ & & \\
\hline
\end{tabular}

Fonte: Elaborado pelos autores com os dados disponíveis por Brayner (1998); Brayner, Barbosa e Silva (2001); Brayner et al. (2003); Silva (2004); Macedo et al. (2007); Silva et al. (2010); Maciel (2015); Régis (2016); Xavier (2017); Xavier et al. (2017); Barcellos et al. (2017) e Régis et al. (2018). 
A análise de metais pesados realizada em sedimentos próximos a viveiro de peixes, na região estuarina do rio Capibaribe, demonstra que a água do viveiro apresentava contaminação devido ao constante lançamento de esgoto, com concentrações elevadas de metais nos sedimentos, principalmente para Zn e Cr. A caracterização da fase mineral dos sedimentos, através do uso de difratometria de raios-X, tornou possível a identificação dos principais argilominerais presentes nos sedimentos, representados por caulinita e ilita. Os metais pesados ( $\mathrm{Zn}, \mathrm{Cr}$, Mn e Fe) foram analisados mediante processos de extração sequencial para determinação da associação química dos mesmos em diversas frações granulométricas e os diferentes estados de oxidação predominantes para cada metal analisado. Os autores inferem os estados de oxidação predominantes de cada elemento analisado a partir de diferentes métodos de extração que fazem distinção entre espécies oxidadas e reduzidas. Os resultados sugeriram que os sedimentos de viveiros de peixes atuam naturalmente como reservatório de metais pesados, mas não indicaram processos de bioacumulação para os peixes (BRAYNER, 1998; BRAYNER; BARBOSA; SILVA, 2001).

O comportamento dos metais pesados no estuário do rio Capibaribe sofrem influência da composição granulométrica dos sedimentos, teor de argilominerais e matéria orgânica. De acordo com Brayner et al. (2003), os sedimentos analisados na região estuarina são compostos por caulinita, ilita, feldspatos, quartzo e hematita, sugerindo que possuem a mesma origem mineralógica. As amostras de sedimentos que possuem uma maior quantidade de argilominerais apresentam maiores concentrações de metais pesados. Entretanto, apenas a determinação granulométrica não é suficiente para analisar tal comportamento, visto que, alguns pontos com predomínio da granulometria arenosa apresentaram alta concentração de metais pesados, sugerindo que outras condições como o tipo de argilomineral, concentração de substâncias húmicas e influência do regime hidrodinâmico afetam o aprisionamento dos metais em sedimentos.

O monitoramento ambiental feito por Silva (2004) e Macedo et al. (2007) foram realizados de forma sazonal para verificar, em função das estações seca e chuvosa, se os metais estão em sua forma móvel (livres na água, sob forma iônica, quando se tornam biodisponíveis) ou imóvel (adsorvidos nos sedimentos). A metodologia usada considerou ainda a coleta de sedimentos superficiais, bem como a coleta de sedimentos depositados durante um período de $24 \mathrm{~h}$, para correlação dos metais pesados retidos no sedimento superficial e no material depositado ao longo de 24 h. A partir disso, determinaram as concentrações de $\mathrm{Cd}, \mathrm{Co}, \mathrm{Cu}, \mathrm{Pb}, \mathrm{Cr}, \mathrm{Fe}, \mathrm{Ni}, \mathrm{Mn}$ e Zn. A concentração de metais pesados no material sedimentado no período de 24 horas demonstrou ser 
relativamente maior que as concentrações obtidas para sedimentos superficiais, indicando uma transferência de metais na área estudada. Concluíram ainda que a variação sazonal é o principal agente associado com a distribuição dos metais pesados no material sedimentado e em sedimentos superficiais na zona estuarina.

Silva et al. (2010) determinaram o background ${ }^{l}$ geoquímico local do estuário, coletando sedimentos na margem do rio Capibaribe na região do Parque dos Manguezais. As concentrações de background obtidas para $\mathrm{Cr}$, Mn e Zn, foram respectivamente 25 mg.kg-1, 160 mg.kg ${ }^{-1}$ e 41 mg.kg-1 Para os autores, tais valores podem ser utilizados para o acompanhamento da evolução da contaminação por metais na região estudada, refletindo a qualidade do ecossistema. No mesmo trabalho, análises de sedimentos na Bacia do Pina indicaram concentrações de cromo, manganês e zinco acima dos valores de referência estabelecidos como background, evidenciando contaminação nos pontos de coleta. Os autores relataram fontes associadas com efluentes domésticos, miniestaleiros e resíduos de empresas galvânicas.

A investigação da qualidade ambiental dos sedimentos estuarinos pode ser feita pela chamada Tríade da Qualidade Ambiental, que consiste em: (i) análise química e investigação de concentração dos contaminantes em sedimentos; (ii) realização de bioensaios para determinação da toxicidade; (iii) análise da população bentônica para analisar os efeitos da contaminação no meio ecológico (CHAPMAN; HOLLERT, 2006). Este método foi utilizado por Maciel (2015) e Régis (2016) em atividades de monitoramento ambiental onde foram avaliadas a contaminação de sedimentos por hidrocarbonetos alifáticos, hidrocarbonetos policíclicos aromáticos, compostos organoestânicos, compostos organoclorados e metais pesados ( $\mathrm{Al}, \mathrm{Ag}, \mathrm{As}, \mathrm{Cd}, \mathrm{Cr}, \mathrm{Cu}, \mathrm{Fe}, \mathrm{Hg}, \mathrm{Ni}$, $\mathrm{Pb}$ e $\mathrm{Zn}$ ), em conjunto com estudos ecotoxicológicos realizados utilizando copépodos (Tisbe biminienses). Estes estudos são os mais completos sobre a qualidade ambiental já realizados no estuário do rio Capibaribe. As concentrações obtidas por Maciel (2015) para metais pesados refletem concentrações abaixo dos limites de segurança determinados por agências internacionais, exceto para cobre, chumbo e zinco. Régis (2016) obteve para $\mathrm{Cd}, \mathrm{Cr}, \mathrm{Cu}, \mathrm{Ni}$ e $\mathrm{Pb}$ concentrações maiores que o "threshold effect level" " (TEL), porém concentrações abaixo do nível do "probably effect level"

\footnotetext{
${ }^{1}$ Concentração relativa para diferenciação entre concentrações naturais de um elemento químico e as concentrações influenciadas pelas atividades antrópicas ao longo do tempo (MATSCHULLAT; OTTENSTEIN; REIMANN, 2000).

${ }^{2}$ Representa a concentração abaixo da qual espera-se que efeitos biológicos adversos ocorram raramente (LONG et al., 1995).

${ }^{3}$ Define o nível acima do qual se espera que os efeitos adversos ocorram com frequência (LONG et al., 1995).
} 
(PEL), enquanto os elementos Ag e Zn encontram-se em níveis superiores ao PEL. A autora destaca que os valores foram obtidos próximo do canal principal do rio Capibaribe sendo influenciado pela comunicação com a Bacia do Pina. Os sedimentos localizados próximo no centro do Recife mostraram-se mais contaminados em comparação com os sedimentos encontrados a montante e a jusante do estuário. Alguns metais, como Ag e Zn, apresentaram concentrações maiores do que os níveis de segurança propostos pelas agências internacionais. As pesquisas realizadas sugeriram que os sedimentos estuarinos estão contaminados por hidrocarbonetos, organoclorados e metais pesados, estando biodisponíveis, ocasionando toxicidade na biota e afetando a população bentônica (MACIEL, 2015; RÉGIS, 2016).

Devido aos processos de instalação e desenvolvimento urbano, a região estuarina do rio Capibaribe passou e passa por diversas mudanças em seu meio biológico, físico e químico. A modificação no padrão de sedimentação, bem como a concentração de metais pesados são associados com a evolução na urbanização ocorrida na Região Metropolitana do Recife. Estudos realizados por Xavier (2017) em dois testemunhos obtidos na região do estuário do rio Capibaribe abrangendo em torno de 200 anos de sedimentação identificaram as atividades antrópicas e seu reflexo na taxa de sedimentação e na concentração de metais pesados no ambiente, tendo sido analisados $\mathrm{Mn}, \mathrm{Fe}, \mathrm{Co}$, $\mathrm{Ni}, \mathrm{Cu}, \mathrm{Zn}, \mathrm{Ga}, \mathrm{As}, \mathrm{Pb}, \mathrm{Ti}, \mathrm{V}, \mathrm{Mg}$ e Al. As análises realizadas no testemunho denominado T2 (com $158 \mathrm{~cm}$ ) demonstraram uma taxa de sedimentação de $0,45 \mathrm{~cm}$ por ano e três facies sedimentares distintas denominadas unidade 1, unidade 2 e unidade 3. Na unidade 1, que alcançou o período compreendido entre os anos de 1812 a 1856, os dados sugerem um ambiente com sedimentação estuarina, predomínio de sedimentos lamosos e concentrações de metais pesados consideradas como naturais no ambiente, ainda com pouco impacto de atividades antrópicas. A unidade 2, abrangendo de 1856 a 1972, evidenciou que o início das obras do Porto do Recife alterou a hidrodinâmica do rio Capibaribe e os processos de sedimentação natural do ambiente estuarino. Há o aumento das porcentagens dos sedimentos arenosos e diminuição dos sedimentos lamosos e matéria orgânica, resultando na diminuição da concentração de metais pesados. A unidade 3, compreendida entre os anos de 1972 a 2012, reflete uma alta taxa de sedimentos finos e matéria orgânica, apresentando elevadas concentrações para os elementos químicos analisados. Em relação ao segundo testemunho (denominado T5, com $178 \mathrm{~cm}$ ), foi possível a subdivisão em quatro unidades diferentes, sendo determinada uma taxa de sedimentação de $0,52 \mathrm{~cm}$ por ano. A unidade 1 , compreendida antes do ano de 1812, possui sedimentos predominantemente finos, altas porcentagens de matéria orgânica e 
concentrações de metais pesados possivelmente de origem geogênica, sugerindo baixa ou nenhuma influência antrópica. Em relação à unidade 2, cobrindo os anos de 1812 a 1937, observou-se aumento de sedimentos arenosos e menor quantidade de sedimentos finos, matéria orgânica e concentrações de metais, decorrentes do processo de expansão urbana e monocultura da cana-de-açúcar. A unidade 3, cobrindo sedimentação entre 1937 e 2004, possui maior quantidade de sedimentos arenosos, menor quantidade de sedimentos finos e matéria orgânica, além de possuir menores concentrações de metais pesados quando comparada às unidades anteriores. Esta unidade representa a intensificação do processo de urbanização ocorrido na Região Metropolitana do Recife. Por fim, a unidade 4 (de 2004 a 2012) apresenta aumento de sedimentos finos, aumento na quantidade de matéria orgânica e na elevação na concentração de metais pesados. Tal comportamento possivelmente está relacionado com o reflorestamento da vegetação de mangue no estuário, mediante ao projeto da Prefeitura do Recife em 2000, alterando o padrão de sedimentação.

Além da evolução dos parâmetros sedimentológicos e geoquímicos na região estuarina do rio Capibaribe, foi possível a partir do estudo da unidade 1 nos dois testemunhos, obter as concentrações de base (background) para os metais pesados, sendo definidos os seguintes valores (em mg.kg $\left.{ }^{-1}\right)$ : Mn (292,2), Fe (2,7\%), Co (10,4), Ni (22,2), Cu (60,8), Zn (105,5), As (106) e Pb $(52,9)$. O estudo também utiliza de fatores geoquímicos para compreender o quanto a área está contaminada (Fator de contaminação) e poluída (Índice de geoacumulação), bem como a possível origem (Fator de enriquecimento) dos metais pesados. Os testemunhos analisados variam de não enriquecidos a moderadamente enriquecidos, não contaminados a moderadamente contaminados e não poluído a moderadamente poluído. As análises realizadas nos sedimentos datados desde o século XIX apresentam modificações de sedimentação, bem como na concentração de metais pesados, além da contaminação por fontes antrópicas o estuário também recebe contribuições de fontes geogênicas, sendo relacionado com a Formação Barreiras devido ser rica em metais pesados ( $\mathrm{Mn}, \mathrm{Fe}, \mathrm{Co}, \mathrm{Cu}, \mathrm{As}$ e Ni) (XAVIER, 2017; BARCELLOS et al., 2017; XAVIER et. al., 2017).

\section{CONSIDERAÇÕES FINAIS}

Neste trabalho, a revisão de literatura evidencia que existe a continuidade e o aumento das emissões antrópicas na região estuarina do rio Capibaribe mediante os últimos 203 anos. As pesquisas realizadas avaliaram os níveis de concentrações de metais pesados ( $\mathrm{Al}, \mathrm{Ag}, \mathrm{As}, \mathrm{Cd}, \mathrm{Co}, \mathrm{Cr}, \mathrm{Cu}, \mathrm{Fe}$, $\mathrm{Hg}, \mathrm{Mn}, \mathrm{Mg}, \mathrm{Ni}, \mathrm{Pb}, \mathrm{Zn}$ ) em sedimentos, além de estudos toxicológicos em copépodos. Além disso, 
foram verificadas a contaminação por HAs, HPAs e OTs, assim como, análise em material sedimentado durante o período de $24 \mathrm{~h}$, originados predominantemente por fontes antrópicas. Embora o estuário receba grande aporte de material antrópico, existe a forte contribuição geogênica vinda da Formação Barreira que influencia na contaminação por metais (particularmente $\mathrm{As}, \mathrm{Pb}$ e Zn).

A diversidade de contaminantes que prejudicam a região estuarina, devido ao constante lançamento de poluentes para o estuário, a ameaça da toxicidade dos compostos, além da pouca eficiência das leis de proteção da região e falta de monitoramento ambiental contribuem no processo de degradação no ecossistema do estuário do rio Capibaribe. Desta forma, torna-se fundamental a realização de mais pesquisas para a avaliação dos contaminantes nestes ambientes, bem como, planos efetivos de manejo ambiental pretendendo a prevenção de futuros danos nos ecossistemas estuarinos.

\section{AGRADECIMENTOS}

À Coordenação de Aperfeiçoamento de Pessoal de Nível Superior (CAPES) pelo apoio científico.

\section{REFERÊNCIAS}

ALMEIDA, C. A.; ROCHA, O. Estudo Comparativo da Qualidade dos Sedimentos dos Reservatórios do Rio Tietê (SP). J. Brazilian Soc. Ecotoxicol., v. 1, p. 141-145, 2006.

AZEVEDO, F. A.; CHASIN, A. A. M. Metais: Gerenciamento da Toxicidade. Editora Atheneu, São Paulo. p. 554, 2003.

BARCELlOS, R. L.; FIGUEIRA, R. C. L.; FRANÇA. E. J.; SCHETTINI, C. A.; XAVIER, D. A. Changes of Estuarine Sedimentation Patterns by Urban Expansion: The Case of Middle Capibaribe Estuary, Northeastern Brazil. International Journal of Geosciences, v. 8, p. 514-535, 2017. https://doi.org/10.4236/ijg.2017.84027

BARROS, H. M. et al. Gerenciamento participativo de estuários e manguezais. Ed. Universitária da UFPE, Recife, p. 252, 2000.

BIRCH, G. F.; OLMOS, M. A. Sediment-bound heavy metals as indicators of human influence and biological risk in coastal water bodies. ICES J. Mar. Sci., v. 65, p. 1407-1413, 2008. https://doi.org/10.1093/icesjms/fsn139

BRAYNER, F. M. M. Determinação de taxas de retenção de metais traço por sedimentos orgânicos em um viveiro de psicultura em área estuarina e urbana. São Carlos: USP/EESC, 1998.

BRAYNER, F. M. M.; BARBOSA, A. M. F.; SILVA, H. K. P. Speciation of Heavy Metals in estuarine sediments in the northeast of Brazil. Environmental Science and Pollution Research, v. 8, n. 4, p. 76-77, 2001. https://doi.org/10.1007/BF02987405 
BRAYNER, F. M. M.; SILVA, H. K. P.; MELO, L. V.; BARBOSA, A. M. F. Behavior of heavy metal in the estuarine area of the Capibaribe river in the Northeast of Brazil. Journal of Physique IV, v. 107, p. 221-225, 2003. https://doi.org/10.1051/jp4:20030283

CAJARAVIlle, M. P.; BEBIANnO, M. J.; BlASCO, J.; PORTE, C.; SARASQUETE, C., VIARENGO, A. The use of biomarkers to assess the impact of pollution in coastal environments of the Iberian Peninsula: a practical approach. Sci. Total Environ., v. 247, p. 295-311, 2000. https://doi.org/10.1016/s0048-9697(99)00499-4

CEVIK, F.; GOKSU, M. Z. L.; DERICI, O. B.; FINDIK, O. An assessment of metal pollution in surface sediments of Seyhan dam by using enrichment factor, geoaccumulation index and statistical analyses. Environmental Monitoring and Assessment,, v. 152, p. 309-317, 2009. https://doi.org/10.1007/s10661-008-0317-3

CHAPMAN, P. M.; HOLLERT, H. Should the Sediment Quality Triad Become a Tetrad, a Pentad, or Possibly even a Hexad. Journal of Soils and Sediments, v. 6, n. 1, p. 4-8, 2006. https://doi.org/10.1065/jss2006.01.152

CICIN-SAIN, B.; KNECHT, R. W. Integrated coastal and ocean management: Concepts and practices. Island Press, Washington, DC, p. 543, 1998.

COELHO, P. A.; TORRES, F. A. Áreas estuarinas de Pernambuco. Trabalhos Oceanográficos da Universidade Federal de Pernambuco, v. 17, p. 67-80, 1982.

CUNHA, P. P; PINTO, J.; DINIS, J. L. Evolução da fisiografia e ocupação antrópica na área estuarina do Rio Mondego e região envolvente (Portugal centro-oeste), desde 1947. Territorium, v. 4, p. 99124, 1997. https://doi.org/10.14195/1647-7723_4_8

DEVESA-REY, R.; DIAZ-FIERROS, F.; BARRAL, M. T. Assessment of enrichment factors and grain size influence on the metal distribution in riverbed sediments (Anllóns River, NW Spain). Environ. Monit. Assess., v. 179, p. 371-388, 2011. https://doi.org/10.1007/s10661-010-1742-7

DUFFUS, J. H. Heavy metals - A meaningless term Pure and Applied Chemistry. Pure Appl. Chem., v. 74, n. 5, p. 793-807, 2002.

FEITOSA, F. D. N.; NASCIMENTO, F. D.; COSTA, K. D. Distribuição espacial e temporal da biomassa fitoplanctônica relacionada com parâmetros hidrológicos na Bacia do Pina (Recife, PE). Trabalhos Oceanográficos da Universidade Federal de Pernambuco, v. 27, n. 2, p. 1-13, 1999.

FRENCH, P. W. Coastal and estuarine management. (Routledge environmental management). New Fetter Lane, London: Routledge, p. 251, 1997.

GU, Y. G.; LIN, Q.; YU, Z. L.; WANG, X. N.; KE, C. L.; NING, J. J. Speciation and risk of heavy metals in sediments and human health implications of heavy metals in edible nekton in Beibu Gulf, China: a case study of Qinzhou Bay. Mar. Pollut. Bull., v. 101, n. 2, p. 852-859, 2015.

LACERDA, L. D.; MOLISANI, M. M.; SENA, D.; MAIA, L. P. Estimating the importance of natural and anthropogenic sources on $\mathrm{N}$ and $\mathrm{P}$ emission to estuaries along the Ceará State Coast NE Brazil. Environ. Monit. Assess., v. 141, p. 149-164, 2008. http://doi.org/10.1016/j.marpolbul.2015.11.019

LIMA, R. F. S.; LOUREIRO, G. L.; MARTINS, I. S. B.; MELO, J. V.; PETTA, R. A. Utilização da espectrometria de fluorescência de raios x para a análise quantitativa de elementos traços em resíduos secos a partir de lixiviados de amostras sólidas. In: XI CONGRESSO BRASILEIRO DE GEOQUÍMICA, 2007, Estância de Atibaia. Anais[...]. Estância de Atibaia: SBGq, 2007. 
LIU, C.; XU, J.; LIU, C.; ZHANG, P.; DAI, M. Heavy metals in the surface sediments in Lanzhou Reach of Yellow River China. Bulletin Environ Contam Toxicol,, v. 82, p. 26-30, 2009. https://doi.org/10.1007/s00128-008-9563-X

LIU, W. X.; LI, X. D.; SHEN, Z. G.; WANG, D. C.; WAI, O. W. H.; LI, Y. S. Multivariate statistical study of heavy metal enrichment in sediments of the Pearl River Estuary. Environmental Pollution, v. 121, p. 377-388, 2003. https://doi.org/10.1016/s0269-7491(02)00234-8

LIU, Z. Y.; PAN, S. M.; SUN, Z. Y.; MA, R. F.; CHEN, L. H.; WANG, Y. L.; WANG, S. A. Heavy metal spatial variability and historical changes in the Yangtze River Estuary and north Jiangsu tidal flat. Mar. Pollut. Bull., v. 98, n. 2, p. 115-129, 2015. https://doi.org/10.1016/j.marpolbul.2015.07.006

LONG, E. R.; MACDONALD, D. D.; SMITH, S. L.; CALDER, F. D. Incidence of adverse biological effects within ranges of chemical concentrations in marine and estuarine sediments. Environ. Manage., v. 19, p. 81-97, 1995.

MACEDO, S. J.; SILVA, H. K. P.; BRAYNER, F. M. M.; DUARTE, M.; BARBOSA, A. M. F. Heavy Metal concentrations in sediments of the Capibaribe River Estuary in the Metropolitan Region of Recife, Pernambuco - Brasil. In: SUSTAINABLE DEVELOPMENT, 2007, Recife. Anais [...]. Recife: WIT, 2007.

MACIEL, D. C.; COSTA, B. V. M.; SANTOS, L. P. S.; SOUZA, J. R. B.; Zanardi-lamardo, E. Avaliação da toxicidade dos sedimentos do sistema estuarino do rio Capibaribe (Pernambuco, Brasil) utilizando o copépodo bentônico Tisbe biminiensis Volkmann Rocco (1973). Tropical Oceanography (Online), v. 43, p. 26-37, 2015. https://doi.org/10.5914/tropocean.v43i1.5882

MACIEL, D. C. Avaliação da contaminação do sistema estuarino do Rio Capibaribe através da tríade da qualidade do sedimento. Dissertação (Mestrado) - Departamento de Oceanografia, Universidade Federal de Pernambuco, Recife, 2015.

MATSCHULlAT, J.; OTTENSTEIN, R.; REIMANN, C. Geochemical background-can we calculate it? Environ Geol., v. 39, n. 9, p. 990-1000, 2000.

MMA - MINISTÉRIO DO MEIO AMBIENTE. Limites da Zona Costeira. Disponível em: http://www.mma.gov.br/gestao-territorial/gerenciamento-costeiro/zona-costeira-e-seusm\%C3\%BAltiplos-usos/caracteristicas-da-zona-costeira.html. Acesso em: 20 mai. 2019.

MORAES, E. C. F.; SZNELWAR, R. B.; FERNíCOLA, N. A. G. G. Manual de toxicologia analítica. São Paulo: Roca, 1991.

NÓBREGA, A. S. C. Fontes de Contaminação no Estuário do Rio Capibaribe, Pernambuco. Trabalho de conclusão de curso (Bacharelado em ciências biológicas) - Universidade Federal de Pernambuco, Recife, 2011.

OLIVEIRA, F. P. Percepção Ambiental e Gestão do Meio Ambiente de Toritama (PE) - Estudo da Percepção de Diferentes Atores Sociais Sobre o Rio Capibaribe. Dissertação (Mestrado em Gestão e Políticas Ambientais) - Universidade federal de Pernambuco, 2007.

OLIVEIRA, T.; BARCELLOS, R. L.; SCHETTINI, C. A. F.; CAMARGO, P. B. Processo sedimentar atual e distribuição da matéria orgânica em um complexo estuarino tropical, Recife, PE, Brasil. Gestão costeira integrada, v. 14, n. 3, p. 399-411, 2014. http://dx.doi.org/10.5894/rgci470 
RÉGIS, C. G. O uso dos náuplios de tisbe biminiensis (copepoda: harpacticoida) na avaliação da toxicidade dos sedimentos do estuário do rio Capibaribe e sua relação com a geoquímica. Dissertação (Mestrado) - Departamento de Oceanografia, Universidade Federal de Pernambuco, Recife, 2016.

RÉGIS, C. G.; SOUZA-SANTOS, L. P.; YOGUI, G. T.; MORAES, A. S.; SCHETTINI, C. A. F. Use of Tisbe biminiensis nauplii in ecotoxicological tests and geochemical analyses to assess the sediment quality of a tropical urban estuary in northeastern Brazil. Marine Pollution Bulletin,, v. 137, p. 4555, 2018. https://doi.org/10.1016/j.marpolbul.2018.10.011

SALOMONS, W.; FORSTNER, V. Metals in hydrocycle. Berlin: Spring Verlag, p. 349, 1984.

SANTOS D. M.; ARAÚJO I. P.; MACHADO E. C. Organotin compounds in the Paranaguá Estuarine Complex, Paraná, Brazil: Evaluation of biological effects, surface sediment, and suspended particulate matter. Mar Pollut Bull., v. 58, p. 1926-1931, 2009. https://doi.org/10.1016/j.marpolbul.2009.09.004

SANTOS, G. T.; BEZERRA-JUNIOR, J. L.; COSTA, K. M. P.; FEITOSA, F. A. N. Dinâmica da biomassa fitoplanctônica e variáveis ambientais em um estuário tropical (Bacia Do Pina, Recife, PE). Revista Brasileira de Engenharia de Pesca, v. 4, n. 1, p. 95-109, 2009.

SCHAEFFER, N. Y.; CINTRÓN, M. G.; ADAIME, R.; CAMARGO, T. Variability of mangrove ecosystems along the Brazilian coast. Estuaries, Springer-Verlag, v. 13, n. 2, p. 204-218, 1990.

SILVA, H. K. P. Concentrações dos metais pesados nos sedimentos do estuário do rio Capibaribe, Região Metropolitana do Recife (RMR) - Pernambuco, Brasil. Dissertação (Mestrado) - Departamento de Oceanografia, Universidade Federal de Pernambuco, 2004.

SILVA, H. K. P.; BRAYNER, F. M. M.; MACEDO, S. J. Avaliação das concentrações de metais traço nos sedimentos do Parque dos Manguezais, Região Metropolitana do Recife (RMR), Pernambuco, Brasil. Tropical Oceanography, v. 38, n. 2, p. 174-180, 2010. https://doi.org/10.5914/tropocean.v38i2.5170

TAVARES, T. M.; CARVALHO, F. M. Avaliação de exposição de populações humanas a metais pesados no ambiente: exemplos do Recôncavo Baiano. Química Nova, v. 15, n. 2, p. 147-154, 1992.

VALLS, M.; LORENZO, V. Exploiting the genetic and biochemical capacities of bacteria for remediation of heavy metal pollution. FEMS Microbiology Reviews, v. 26, p. 327-338, 2002. https://doi.org/10.1111/j.1574-6976.2002.tb00618.x

VERONEZ JÚNIOR; BASTOS, P.; CARDOSO, A.; SILVA, V. Morfologia e distribuição sedimentar em um sistema estuarino tropical: Baía de Vitória, ES. Revista Brasileira de Geofísica, v. 27, n. 4, p. 609-624, 2009. http://dx.doi.org/10.1590/S0102-261X2009000400006

WANG, Z.H.; GUO, X.; ZHANG, K.; LU, X. X. Environmental changes in Jiaozhou Bay of northern China during the past 90 years using metals and biogenic elements. J. Environ. Sci., v. 53, p. 301312, 2017. https://doi.org/10.1016/j.jes.2016.06.002

WILLIAMS, T. P.; BUBB, J. M.; LESTER, J. N. Metal accumulation Salt Marsh Environments: A review. Marine Pollution Bulletin, v. 28, n. 5, p. 277-290, 1994. DOI: 10.2307/4494133

WOOD, J. M. Biological cycles for toxic elements in the accumulation Salt Marsh Environments: A review. Marine Pollution bulletin, v. 28, n. 5, p. 277-290, 1974. 
XAVIER, A. D. Sedimentação Recente do Médio e Baixo Estuário do Rio Capibaribe - Recife - Pernambuco. Tese (Doutorado) - Departamento de Oceanografia, Universidade Federal de Pernambuco, Recife, 2017.

XAVIER, D. A.; SCHETTINI, C. A.; FRANÇA, E. J.; FIGUEIRA, R. C.; BARCELlOS, R. L. Determination of geochemical background values on a tropical estuarine system in a densely urban area. Case study: Capibaribe estuary, Northeastern Brazil. Marine Pollution Bulletin, v. 123, n. 2, p. 381-386, 2017. https://doi.org/10.1016/j.marpolbul.2017.09.007

YANG, H.; ZHUO, S.; XUE, B.; ZHANG, C.; LIU, W. Distribution, historical trends and inventories of polychlorinated biphenyls in sediments from Yangtze River Estuary and adjacent East China Sea. Environmental Pollution, v. 169, n. 1, p. 20-26, 2012. https://doi.org/10.1016/j.envpol.2012.05.003 